\title{
An Optimized Approach on Link Stability with Load Balancing in MANET using Balanced Reliable Shortest Route AOMDV (BRSR_AOMDV)
}

\author{
D. Maheshwari ${ }^{1 *}$ and R. Nedunchezhian ${ }^{2}$ \\ 'Bharathiar University, Coimbatore - 641046, Tamil Nadu, India; maheshgkrish@gmail.com \\ 2Kalaignar Karunanidhi Institute of Technology, Coimbatore -641402, Tamil Nadu, India; rajuchezhian@gmail.com
}

\begin{abstract}
Objectives: MANET consistency depends on the robustness of the load balancing and link between the mobile nodes of the network. This paper has been evaluated the reliability for defective nodes (transceivers) to find the average queue length and signal interference noise ratio. In order to increase the load balancing and produce high throughput. Methods/ Analysis: This paper proposes Balanced Reliable Shortest Route AOMDV (BRSR-AOMDV) a technique which handles both load balancing and link stability based on the average queue length computation and the signal strength of the each reachable links. This proposed approach uses two phases, one for discovering the path with least queue size and in the second phase among the selected paths with short queue size the path with high link stability is considered as optimal path for traversing packet from the selected source to destination nodes. Findings: The method is illustrated with an application and some imperative results are also presented. The performance is done based on different metrics for load balancing as well as link stability and the proposed method performs better than the AODV. Conclusion: BRSR-AOMDV achieves good packet delivery ratio, more network lifetime while attaining low delay overhead than the existing scheme AODV scheme.
\end{abstract}

Keywords: Fault Tolerance, Load balancing, Link Stability, MANET, Queue size

\section{Introduction}

Mobile Ad hoc Network (MANET) is self-organized wireless and mobile networks. Due to mobility the topology of the network changed. Each mobile node in an ad hoc network moves by chance and acts as both a router and a host ${ }^{1}$. However, due dynamic topology centralized management security routing in MANET is the challenging task.

Fault-tolerant of a network to deliver the data successfully within the time period when some node or link failure occurs. A network fault-tolerant method can offer reliable Quality of Service (QoS) guarantee.
Since it is difficult to predict and hold various network attacks. Normally the fault-tolerant mechanisms focus on keeping up maintaining the transmission of important information the network cannot completely withstand and eliminate faults. Packet loss due to network faults will cause great damage in urgent situation events. Therefore, it is essential for WMNs to supply fault-tolerant functions to ensure the victorious transmission of significant data in a judicious manner.

AOMDV (Ad Hoc On-demand, multipath distance vector routing protocol) is a routing protocol specially designed for MANET environment. On demand multipath protocols discover multiple paths between the

${ }^{*}$ Author for correspondence 
source and the destination in a single route discovery. So, a new route discovery is needed only when all these paths fail $^{4}$. AOMDV extends the AODV protocol to discover multiple paths between the source and the destination in every route discovery. Multiple paths so computed are guaranteed to beloop-free and disjoint. AOMDV has three novel aspects compared to other on-demand multipath protocols. First, it does not have high inter-nodal coordination overheads. Second, it ensures disjointness of alternate routes via distributed computation without the use of source routing. Finally, AOMDV computes alternate paths with minimal additional overhead over AODV; it does this by exploiting already available alternate path routing information as much as possible. In BRSR_AOMDV route table entry has a new field AQL. Besides a route list is used in BRSR_AOMDV to store additional information for each alternate path including: Next hop, last hop, hop count, and expiration timeout and AQL. The AQL field is used the find a average queue length of all available path.

This paper handles two major issues in BRSR_AOMDV they are load balancing and link stability. A critical part of the finest promising network is the load balancing. For illustration, job completion becomes difficult, if massive load is given to the nodes with less processing capabilities and which do not have any means to share the load ${ }^{11}$. There is a chance of load disproportion due to that the computing power of the systems are non-uniform it means few nodes may be idle and few will be overloaded. The second major issue in MANET is nodes are moving randomly without any centralized administration in case if these nodes are not having reliable stability of neighbor nodes, links, and paths from source to destination, it will suffer more loss in link. This proposed work introduces an innovative technique to handle both these issues.

\section{Related Works}

In Join-Idle-Queue (JIQ) used for distributed load balancing in high volume system was proposed by ${ }^{21}$. They also introduced another mechanism for random dispatching of packets using Equal-Cost-Multipath. This approach reduces the communication cost considerably. This is applied only for web services and not in mobile ad hoc networks.

In their work ${ }^{12}$ on Ad hoc On-demand Trusted-path Distance Vector protocol (AOTDV) which is used to select multiple loop free paths in one route discovery process, the selection of shortest path based on two approach that is hop count and trust values. Author in ${ }^{17}$ projected an algorithm for selection of backup path with high reliability. This algorithm estimate link reliability with the help of link expiration time between two nodes. It also finds the routes simultaneously for node-disjoint and link-disjoint path. Author in ${ }^{3}$ designed a framework on anonymous multipath routing protocol based on secret sharing, to detect the activate attack using hash function.

Author in ${ }^{9}$ introduced a novel method on Multipath OLSR; multiple paths obtained using Multipath Dijkstra Algorithm. This algorithm aims to create flexibility and extensibility of path by using different link metrics and cost functions. Author in ${ }^{2}$ proposed a novel approach that enhanced the reliability under path failures and load balancing. This approach identified the disjointpath using mechanism of Stream Control Transmission Protocol and Dynamic Source Routing Protocol.

Author in ${ }^{16}$ adapted Markov Model to predict the link connectivity based on link duration and estimate the settling time for node movement. Author in ${ }^{14}$ proposed Route Stability based QOS Routing Protocol which computes link stability and route stability based on received signal strengths. The work given in ${ }^{15}$ was based on Dynamic Congestion Detection and control Routing (DCDR) method which is proposed to avoid congestion to estimate average queue length at the node level, based on this to identify the congestion level of the node and send warning message to neighbour nodes. Author in ${ }^{4}$ presented a new approach to apply the queue to a power saving in wireless sensor networks and also apply N-Policy of discrete time queue with disasters. The work given in ${ }^{5}$ anticipated a scalable and reactive data replication which increases data availability with the help of frequently used items. SCALAR achieves fault-tolerance and load balancing; it is applied in virtual backbone structure to perform data lookup and replication operation. Author in ${ }^{19}$ presented Channel -Aware AOMDV which is used to select the stable link based on a routing metric channel average non-fading duration. Identification of the link stability using multi-objective approach, that is average queuing delay, energy cost and link lifetime is discussed in $^{20}$.

The work given in ${ }^{10}$ path duration is a design parameter, which establishes a relationship between path duration includes node density, velocity of nodes, transmission range and number of hops. Author in ${ }^{11}$ 
proposed a fault-tolerant cluster-based QOS, the link failure was evaluated using failure recovery time, packet dropped, throughput and flow bandwidth. Author in ${ }^{6}$ proposed LAER Link-stability and Energy aware Routing Protocols. This approach satisfies bi-objective optimization link stability and minimum drain rate energy consumption ${ }^{6}$.

Author in ${ }^{18}$ proposed reliable paths based on the number of links; also introduce prediction base link availability estimation to identify the link reliability. The work given in ${ }^{1}$ takes two metrics to find the node lifetime and link lifetime that they represent is energy drain rate and mobility estimation rate of nodes respectively. It was implemented in Dynamic Source Routing (DSR) which used two mechanisms such as lifetime-prediction routing and signal-stability-based adaptive routing. The work given in ${ }^{8}$ improves routing efficiency to select the stable path, which leads to reduced latency and overhead. The work proposed was applied on Random Direction Model through this to selects an optimal path in terms of path availability.

Author in ${ }^{22}$ proposed to identify the link stability using multi-objective approach, that is average queuing delay, energy cost and link lifetime.

Author in ${ }^{23}$ proposed to develop a new multipath load sharing algorithm using channels sensing, node energy level analysis and malicious node detection. The selection of best path based on packet count.

All the existing work discussed above either rely load balancing or link stability. None of them integrated or taken in to the account both the problems. So this proposed work aims dealing with both load balancing and link stability in AOMDV.

\section{The Proposed Process}

This paper proposes an Optimal Balanced Reliable Shortest Routing technique is for the establishment of effective routing of the packets in case of load balancing and link stability. This technique minimizes the packet drop and delay while increasing the packet delivery ratio. The work ${ }^{13}$ aims at overcoming two critics in AOMDV. They are efficient load balancing and maintaining the link stability. The work consist of two phases, in the first phase load balancing is handled using the computation of average queue length of each reachable neighbour nodes using instantaneous queue length and weight factor of each nodes. The path with minimum queue lengths is selected as the potential routes for packet traversal to the destination node. In the phase 2 link stability of each selected routes from the phase 1 is computed based on the signal to interference ratio. The route which has the SIR value less than the defined threshold value is considered as the best route for traversing the packets to reach the destination. Here the threshold value is set to 15 to $17 \mathrm{db}$. The main objective of this work is determining optimized path from source to destination in AOMDV. First finding average queue length of each reachable path from the source to destination node in order to overcome load imbalance is performed which results in the selection of set of paths which have minimized queue length. Next the best stabilized link is determined and finally the path which satisfies both these criteria is chosen as the optimized route for packet transfer in BRSR-AOMDV.

The below Figure 1. From the available routes path with minimum queue lengths are selected and represented using R1, R2 and R3. The selection process is based on the size of AQL and main objective is to determine the minimum load balancing path. The route $\mathrm{R} 1$ has the minimum AQL with comparing to R2 and R3. The R2 has one single node as medium Queue length whereas the R3 consist of two medium queue lengths. Finally we opt to proceed with Rout R1 and R2 for finding better link stability. From the selected paths of phase 1 the link stability with minimum threshold is R1 and it is selected as optimal BSSR for packet traversal from the Source $\mathrm{S}$ to Destination D.

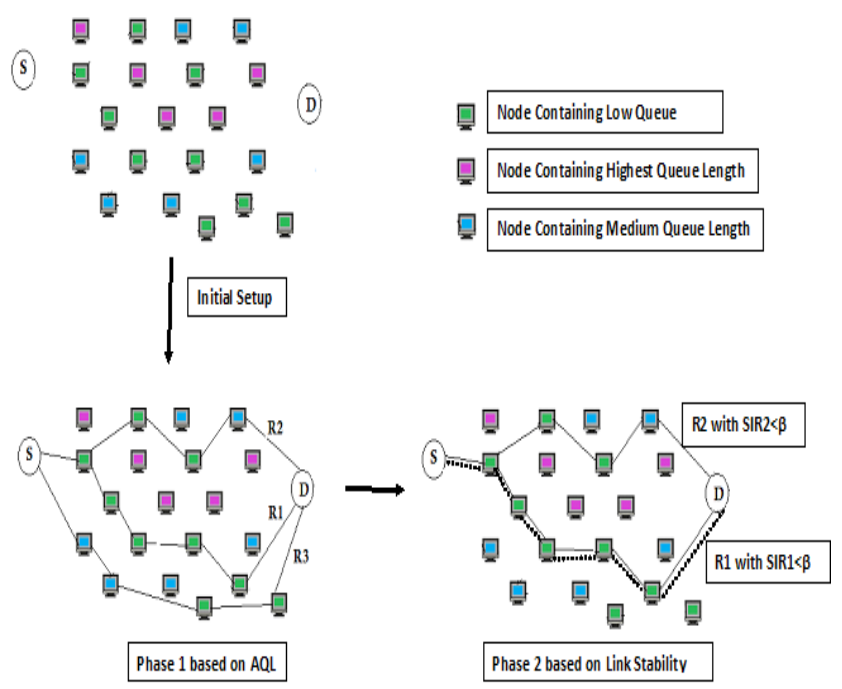

Figure 1. Optimal route selection based on load balancing and link stability. 


\begin{tabular}{|c|}
\hline BRSR_AOMDV Algorithm \\
\hline Input: Number of Nodes \\
\hline Output : Optimal Route \\
\hline $\begin{array}{l}I^{*} \text { This algorithm is to find an optimal route using load bal- } \\
\text { ancing and link stability. The following parameters are used } \\
\text { S ßSource_Node } \\
\text { DßDestination_Node } \\
\mathrm{N}_{\mathrm{i}} \text { ßIntermediate_Node } \\
\text { Nß number of nodes }\end{array}$ \\
\hline $\begin{array}{l}\text { Begin } \\
\text { Step 1: Initiate Route Discovery Process using } \\
\text { BRSR_AOMDV, to find all available path between } \\
\text { source } S \text { to destination } D \text {. Total number of available } \\
\text { is n_path. } \\
\text { Step 2: call load_balance( }) \\
\text { Step 3: Choose the optimal path }\left(\mathrm{S}_{\text {path }}\right) \text { to traverse } \\
\text { the packet } \\
\text { End }\end{array}$ \\
\hline Find Average_Queue_Lenth of all available path \\
\hline Input : Number of nodes n, Number of paths n_path \\
\hline Output : Path with minimum load \\
\hline $\begin{array}{l}l^{*} \text { This procedure is to find the Average_Queue_Length } \\
\text { (AQL) for all available path. The following parameters are } \\
\text { used } \\
\text { Wf } ß 0.002 \\
Q_{\text {thres }} ß \text { Constant_Value } \\
\text { AQL } \\
\text { IQLath_id) } B \text { Average_Queue_Length } \\
\text { Path }{ }_{\text {no }} ß 0 \\
\text { Npß number of packets } \\
1 \text { B packet arrival per unit time } \\
S_{\text {path }} B \text { Selected_path } \\
\star \text { I }\end{array}$ \\
\hline $\begin{array}{l}\text { Begin } \\
\text { Step } 1 \text { IPR } \beta N_{P}{ }^{*} 1 \\
\text { Step } 2 \text { :set Path }{ }_{\text {no }} \text { ß Path_Id }{ }_{i}\end{array}$ \\
\hline $\begin{array}{l}\text { Step } 3 \text { : While }(\text { n_path }>0) \\
\text { (n) }\end{array}$ \\
\hline $\mathrm{AQL}_{\text {Pathno }}=(1-\mathrm{wf})^{\star} \mathrm{AQL}_{\text {Pathno }}+\left(\mathrm{IQL}^{*} \mathrm{wf}\right)$ \\
\hline 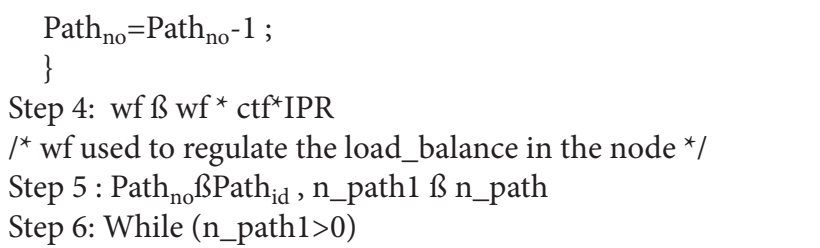 \\
\hline
\end{tabular}

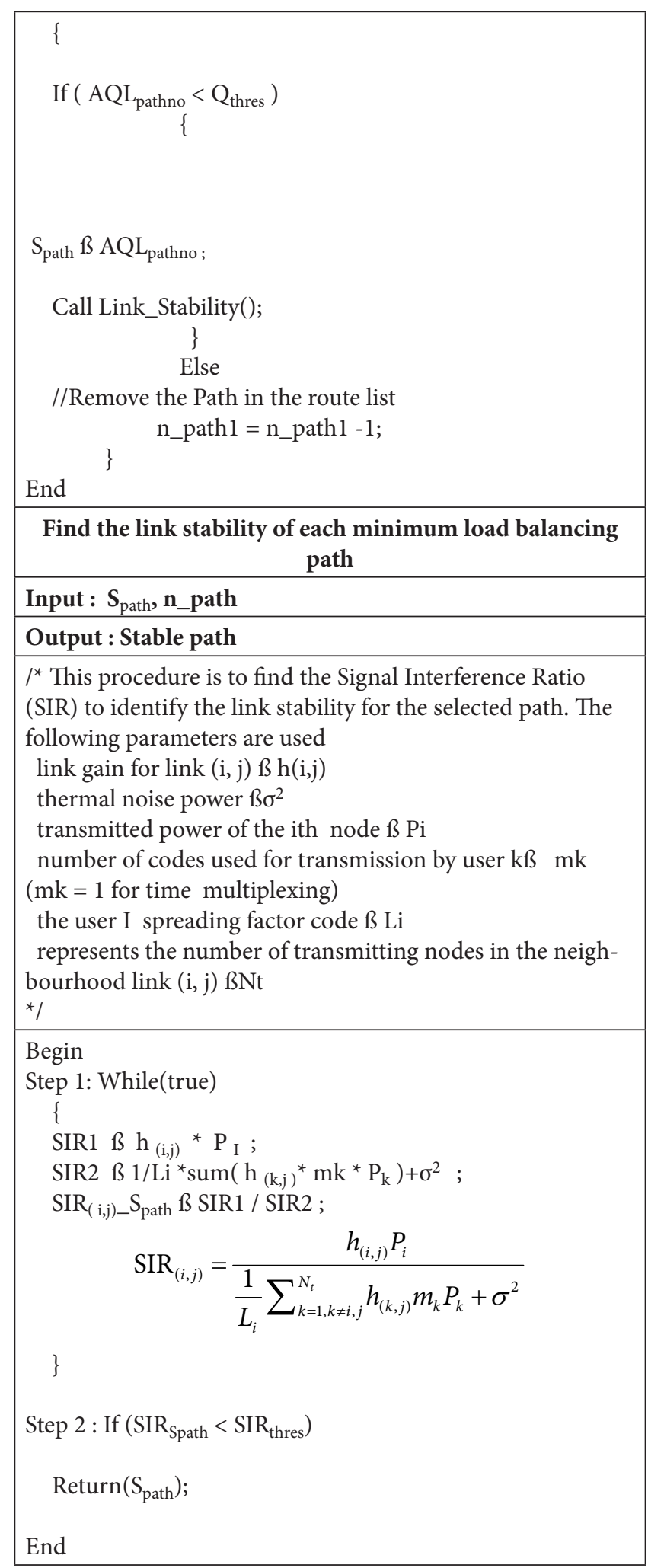




\subsection{Stability of Neighbour Nodes}

There are two parameters taken in to the consideration of neighbour nodes stability. i.e., Mobility, Link loss Path mobility is measured using packets as follows:

Suppose if there are two nodes A and B then the mobility of node PQ

$$
P Q_{\text {mob }}=\frac{\text { Num of packets measured from } P \text { to } Q}{\text { Num of packets measured from } Q \text { to } P}
$$

The node link loss can be measured by using Signal to Noise Ratio. It can be measured by using ${ }^{7}$ Bit Error Rate (BER) which is related to SNR as follows: Let F be the fading in the channel, given by

$$
F=\frac{P_{t r}}{d^{2} j}
$$

Fading can be also represented as the difference between transmitted and received power of source and destination mobile nodes.

$$
F=P_{t r}-P_{r r}
$$

Signal to Noise Ratio (SNR) is given as ratio of transmitted power to the noise power. It is given by

$$
S N R=\frac{P_{t r}}{N_{0}}
$$

If channel is fading based, Noise power is also the fading power. So the Signal to Noise Ratio (SNR) in $\mathrm{db}$ can be represented as:

$$
\begin{gathered}
5 N R=10 \log \left(\frac{P_{t r}}{F}\right) \\
S N R=\frac{P_{t r}}{P_{t r}-P_{r r}}
\end{gathered}
$$

If we take it in to non logarithmic scale,

When the noise power N0 or fading is more, Signal to Noise Ratio decreases and Bit Error Rate also decreases. This relationship is represented by following equation. Hence Bit error rate

Where, $\mathrm{J}=1$

$$
\begin{aligned}
& P_{b} \alpha \frac{1}{S N R} \\
& P_{b}=\frac{J}{S N R} \\
& P_{b}=\frac{1}{S N R}
\end{aligned}
$$

From the link loss with signal to noise ratio and mobility of the nodes, the stability of neighbour nodes is easily measured. The neighbour node stability is estimated by the combination of mobility and link loss of the node.

\subsection{Stability of Path in Whole Network}

Similarly, if there are 'n' numbers of nodes then Mobility of path PS is measured as follows:

Mob of path $P=$ Mob of $P Q{ }^{*}$ Mob of $Q R{ }^{\star}$ Mob of $R S$

And the link loss of the path AD is measured as follows:

Link loss of path $P S=$ link loss of $P Q+$ link loss of $Q R+$ link loss of $R S$

Therefore, by using the two parameters the mobility and link loss, the stability of the path is measured as follows

$$
S_{p}=\frac{M_{p}-L L_{p}}{H_{c}}
$$

Where $\mathrm{S}_{\mathrm{p}}=$ Stabilitypath,

$\mathrm{M}_{\mathrm{P}}=$ mobilitypath,

$\mathrm{LL}_{\mathrm{p}}=$ link loss path,

$\mathrm{H}_{\mathrm{c}}=$ Number of hop count

We also proposed stability of path from stability of link by following calculations. When the distance between two nodes becomes larger than the transmission range the nodes will be disconnected. For transmission range $T_{r}$ link stability $\mathrm{L}_{\mathrm{sb}}$ between any two nodes overtime period $\mathrm{t}$ can be calculated by:

$$
\begin{aligned}
L_{s b}= & \frac{T_{r}}{\sqrt{\left\{\left(p_{1}-p_{2}\right)+t\left(n_{1} \cos \theta_{1}+n_{2} \cos \theta_{2}\right)\right\}^{2}}} \\
& +\left\{\left(q_{1}-q_{2}\right)+t\left(n_{1} \sin \theta_{1}+n_{2} \sin \theta_{2}\right)\right\}^{2}
\end{aligned}
$$

Note that $\mathrm{L}_{\mathrm{sb}}$ is the link stability of individual links between any two nodes and for a path it is a concave parameter and it is same as the minimum link stability along the path. For a path from source to destination path stability $\mathrm{P}_{\mathrm{sb}}$ is given by,

$$
P_{s b}=\operatorname{Min}\left(L_{s b}(1), L_{s b}(2), L_{s b}(3) \ldots \ldots L_{s b}(N)\right)
$$

Where $1,2,3 \ldots \mathrm{N}$ is the number of links along the path.

\section{Experimental Result}

\subsection{Simulation Setup}

In order to evaluate BRSR-AOMDV, we have compared its performance with AODV using several performance metrics. We have used NS-2 as the simulation environment. In our scenario two phases used to find the 
balanced reliable shortest route to solve the link failure problem and balance the load of the route. The area in which the nodes are spread is 1000 X 1000 meters and there are 100 nodes which can move in a range of 250 meters in random directions. In this work we adapted CBR traffic model and each node uses the IEEE802.11 protocol in its MAC layer and the total simulation time is 10 minutes and the packet size is which is to be delivered to single destination is 512 bytes.

Table 1. Simulation setup

\begin{tabular}{|c|c|}
\hline Parameter & Value \\
\hline Transmission Range & $250 \mathrm{~m}$ \\
\hline Topology size & $1000 \mathrm{~m} \mathrm{X} \mathrm{1000m}$ \\
\hline No. of nodes & 100 \\
\hline No. of destination & 1 \\
\hline Traffic type & CBR \\
\hline Packet size & 512 byte \\
\hline MAC layer & 802.11 \\
\hline Bandwidth & 2 Mbps \\
\hline Node placement & Random \\
\hline Simulation Time & 10 minutes \\
\hline Routing Protocol & AODV, AOMDV \\
\hline Queue Size & 100 \\
\hline
\end{tabular}

\subsection{Performance Metric}

\subsubsection{Quеие Size Vs Delay}

The chart above displays the queue size vs. Time delay in dispatching the data to the neighboring nodes. The performance of the proposed system was compared with the AODV and the result shows that the proposed technique better performs by taking less time delay comparing to the existing technique this is possible due

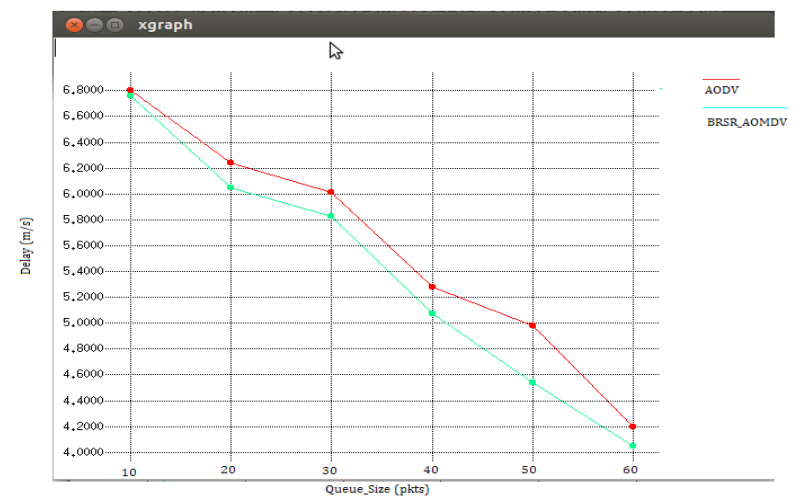

Figure 2. Queue size vs delay. to the load balancing based on usage of average queue length.

\subsubsection{Link Stability Vs Propagation Distance}

Determining the life time of the path statistic were performed for several propagation distance in case of AODV and our proposed method BRSR-AOMDV protocols, whose nature of packet variation becomes as in Figure 3. The proposed approach perform better when the propagation distance of data packets increases because the path selected for transferring the data packets is with the path which has less load while lead to more stable link path from source to destination. BRSR-AOMDV is superior to AODV especially when the $80 \%$ node's propagation distance begins to rise.

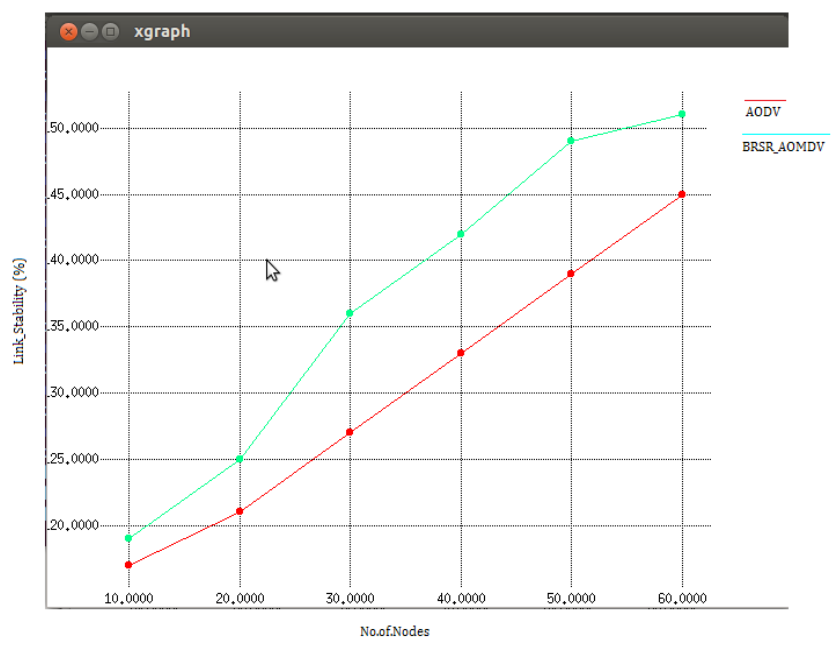

Figure 3. Link stability vs propagation distance.

\subsubsection{Number of Nodes Vs Link Stability}

The performance comparison shows that when the number of nodes increases the link stability ratio also increase it is because each time a path contains more no of nodes the energy loss can be compensated while finding the best short queue size in the overall network which is reachable for source to destination. In this result the proposed work increase link stability more than $60 \%$ compared to AODV.

\subsubsection{Queue Size Vs Throughput}

Figure 5 show the results of while there is increase in the queue size for the throughput 35, 40, 45, 90 for the nodes ranges from 10, 20, 30...60 scenario. Clearly our Proposed BSSR AOMDV scheme achieves $40 \%$ network 


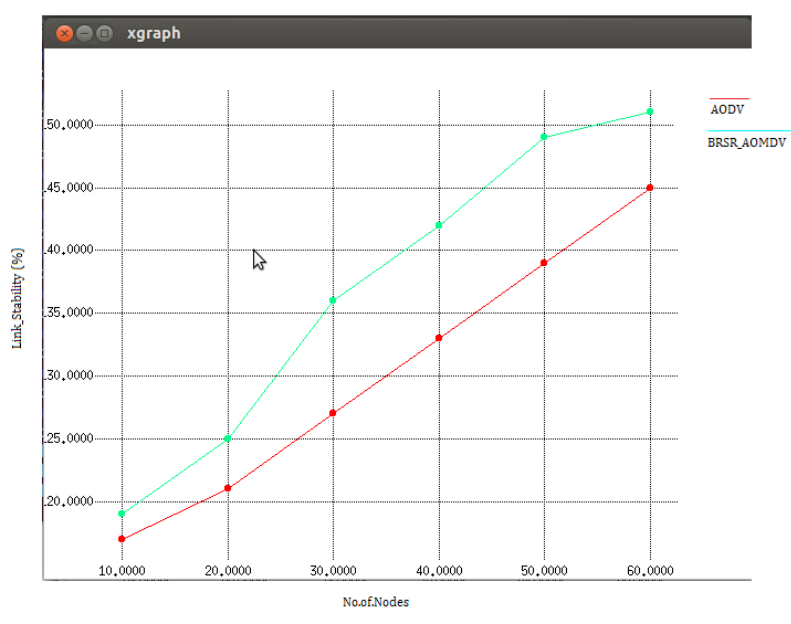

Figure 4. No of nodes vs link stability.

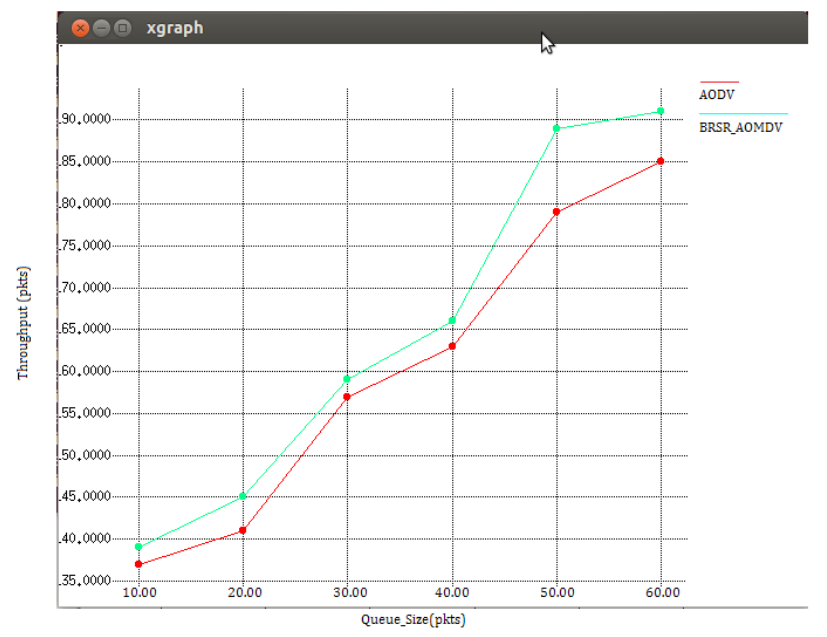

Figure 5. Queue size vs throughput.

throughput than the AODV scheme since it has both predicting stability features.

\subsubsection{Queue Size Vs Packet Delivery Ratio}

Figure 6 show the results of packet delivery ratio for the stability queue size $10,20 \ldots 60$ for the 100 nodes scenario. Clearly our BRSR-AOMDV scheme achieves $80 \%$ delivery ratio than the AODV scheme since it has both reliability and stability features.

\subsubsection{Queue Size Vs Simulation Time}

The performance of the Queue size vs simulation time shows that the proposed method takes more time when the queue size increase because the load balancing and the link stability has to be determined before choosing

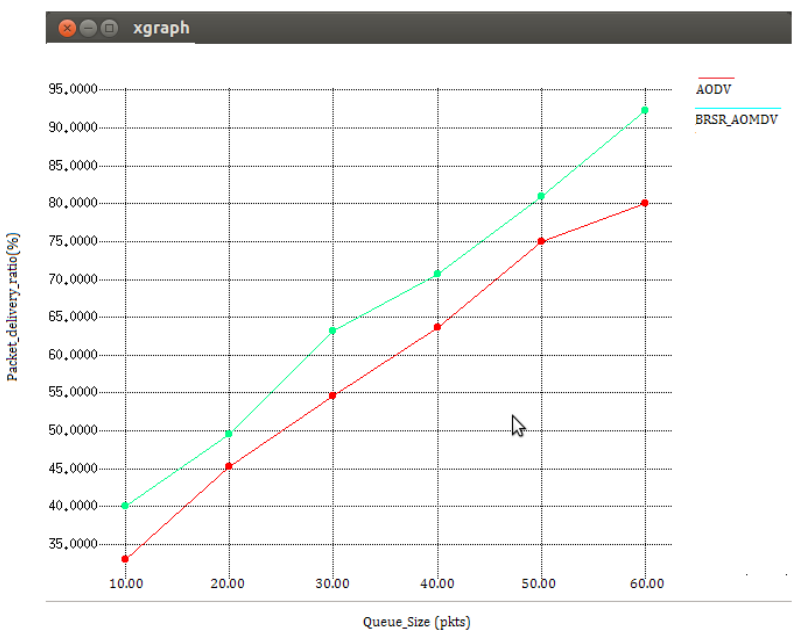

Figure 6. Queue size vs packet_delivery_ratio.

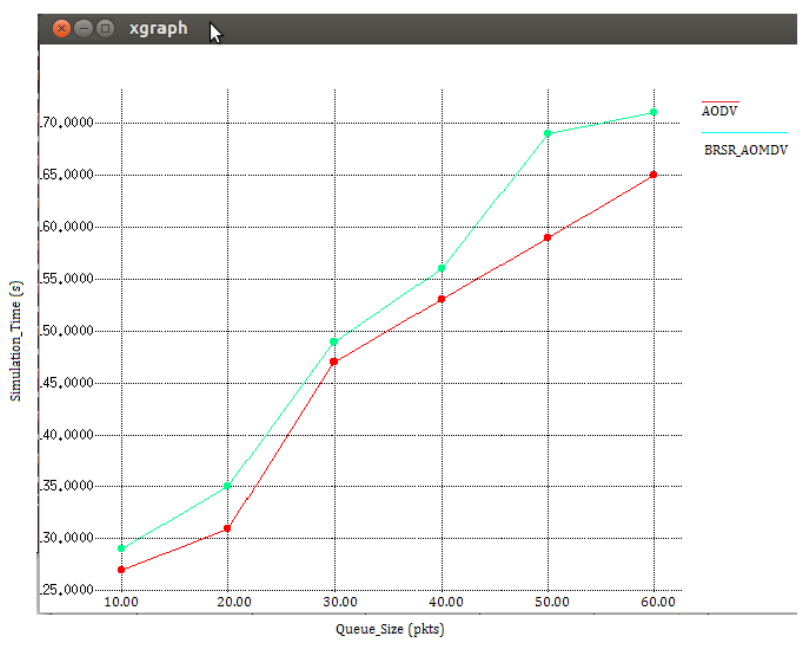

Figure 7. Queue size vs simulation time.

the path but in case of the AODV the option of selection optimal path is missing because it holds simple strategy to select the path which leads $50 \%$ to packet drops and the link failure.

\section{Conclusion}

In this paper, we have developed a prediction based stability scheme with stability models which attains stability in link, path and neighbour nodes. In the first phase of the scheme, stability of neighbour nodes is achieved using Load balancing. The load balancing is obtained by determining the average queue size of each reachable neighbour node from source to destination. The path with least queue size is selected for optimal 
traversal of data packets. In second phase, stability of path is achieved. It uses two factors called mobility factor and link stability. The stability of total mobile nodes is reached using the threshold signal strength value. In this we predicted the network lifetime of the whole network. By simulation results we have shown that the BRSRAOMDV achieves good packet delivery ratio, more network lifetime while attaining low delay overhead than the existing scheme AODV scheme while varying the number of nodes, node speed, throughput and stability weight

\section{References}

1. Nayebi A, Sarbazi-Azad H. Analysis of link lifetime in wireless mobile networks. Elsevier Journal of Ad Hoc Networks. 2012 Sep; 10(7):1221-37.

2. Argyriou A, Madisetti V. Using a new protocol to enhance path reliability and realize load balancing in mobile ad hoc networks. Elsevier Journal Ad Hoc Network. 2006 Jan; 4(1):60-74.

3. Siguang C, Meng W. Anonymous multipath routing protocol based on secret sharing in mobile ad hoc networks. Springer Journal of Systems Engineering and Electronics. 2011; 22(3):519-27.

4. Lee DH, Yang WS. The N-policy of a discrete time Geo/G/1 queue with disasters and its application to wireless sensor networks. Elsevier Journal Applied Mathematical Modelling. 2013 Dec; 37(23):9722-31.

5. Atsan E, Ozkasap O. SCALAR: Scalable data lookup and replication protocol for mobile ad hoc networks. Elsevier Journal Computer Networks. 2013 Dec; 57(17):3654-72.

6. De Rango F, Guerriero F, Fazio P. Link-stability and energy aware routing protocol in distributed wireless networks. IEEE Transactions on Parallel and Distributed Systems. 2012 Apr; 23(4):713-26.

7. [7] Geetha Nair and Dr.N.J.R.Muniraj, "Prediction based Link Stability Scheme for Mobile Ad Hoc Networks", IJCSI International Journal of Computer Science, 2012 November,9(6),401-408.

8. Carofiglio G, Chiasserini C-F, Garetto M, Leonardi E. Route stability in MANETs under the random direction mobility model. IEEE Transactions on Mobile Computing. 2009 Sep; 8(9):1167-79.

9. Yi J, Adnane A, David S, Parrein B. Multipath optimized link state routing for mobile ad hoc networks. Elsevier Journal Ad Hoc Network. 2011 Jan; 9(1):28-47.
10. Namuduri K, Pendse $\mathrm{R}$ Analytical estimation of path duration in mobile ad hoc networks. IEEE Sensors Journal. 2012 Jun; 12(6):1828-35.

11. Llewellyn LC, Hopkinson KM, Graham SR. Distributed faulttolerant quality of wireless networks. IEEE Transactions on Mobile Computing. 2011 Feb; 10(2):175-90.

12. Li X, Jia Z, Zhang P, Zhang R. Trust-based on-demand multipath routing in mobile ad hoc networks. IETInformation Security. 2013 Dec; 4(4):212-32.

13. Marina MK, Das SR. Ad hoc on-demand multipath distance vector routing. Wireless Communications and Mobile Computing. 2006; 6:969-88.

14. Sarma N, Nandi S. Route stability based QoS routing in mobile ad hoc networks. Springer Wireless Personal Communication. 2009 Mar; 54:203-24.

15. Senthilkumaran T, Sankaranarayanan V. Dynamic congestion detection and control routing in ad hoc networks. Journal of King Saud University - Computer and Information Sciences. 2012 May; 25:25-34.

16. Hwang SK, Kim DS. Markov model of link connectivity in mobile ad hoc networks. Springer Journal of Telecommunication System. 2007 Feb; 34:51-8.

17. Sheikhan M, Hemmati E. High reliable disjoint path set selection in mobile ad-hoc network using hop field neural network. IET-Communications. 2011; 5(11):1566-76.

18. Jiang S, He D, Rao J. A prediction-based link availability estimation for routing metrics in MANETs. IEEE/ACM Transactions on Networking. 2005 Dec; 13(6):1302-12.

19. Chen X, Jones HM, Jayalath D. Channel-aware routing in MANETs with route handoff. IEEE Transactions on Mobile Computing. 2011 Jan; 10(1):108-12.

20. Zhang XM, Zou FF, Wang EB, Sung DK. Exploring the dynamic nature of mobile nodes for predicting route lifetime in mobile ad hoc networks. IEEE Transactions on Vehicular Technology. 2010 Mar; 59(3):1567-72.

21. Lua Y, Xie Q, Kliot G, Geller A, Larus JR, Greenberg A. Join-Idle-Queue: A novel load balancing algorithm for dynamically scalable web services. Elsevier Journal Performance Evaluation. 2011 Aug; 68:1056-71.

22. Guo Z, Malakooti S, Sheikh S, Al-Najjar C, Malakooti B. Multi-objective OLSR for proactive routing in MANET with delay, energy, and link lifetime predictions. Elsevier Journal of Applied Mathematical Modelling. 2010 Sep; 35:1413-26.

23. Jagadeesan D, Narayanan S, Asha G. Efficient load sharing using multipath channel awareness routing in mobile ad hoc networks. Indian Journal of Science and Technology. 2015 Jul; 8(15):67729-33. 